\title{
EFEITO DE BORDA COMO FONTE DA HETEROGENEIDADE DO COMPONENTE ARBÓREO EM UMA FLORESTA COM ARAUCÁRIAS NO SUL DO BRASIL
}

\author{
EDGE EFFECT AS SOURCE OF HETEROGENEITY OF THE TREE COMPONENT IN AN \\ ARAUCARIA FOREST IN SOUTHERN BRAZIL
}

\author{
Francieli Pscheidt ${ }^{1}$ Pedro Higuchi ${ }^{2}$ Ana Carolina da Silva ${ }^{2}$ Tássio Dresch Rech ${ }^{3}$ Bruna Salami ${ }^{1}$ \\ Tiago de Souza Ferreira ${ }^{1}$ Marcelo Bonazza ${ }^{4}$ Marco Antonio Bento ${ }^{4}$
}

\begin{abstract}
RESUMO
Objetivou-se avaliar as alterações na organização de uma comunidade arbórea causadas pelo efeito de borda em um remanescente de Floresta com Araucárias no sul do Brasil. Foram instaladas cinco transeções de 100 x $20 \mathrm{~m}$ na área de estudo, perpendiculares às bordas florestais, subdivididas em dez parcelas. Foi definido como setor borda a distância de $0-50 \mathrm{~m}$ e o interior, após $50 \mathrm{~m}$. Todas as árvores com circunferência na altura do peito $\geq 15,7 \mathrm{~cm}$ foram mensuradas, identificadas e classificadas em guildas de regeneração. Os setores foram comparados por meio do teste t, para variáveis com distribuição normal, teste de MannWhitney, para distribuições não normais, rarefação, para a riqueza, e teste de qui-quadrado aplicado a uma tabela de contingência, para verificar a distribuição dos indivíduos das diferentes guildas de regeneração. A setorização em função da borda e interior representou uma importante fonte de heterogeneidade florestal, uma vez que a parte mais marginal do fragmento caracterizou-se por apresentar um componente arbóreo de maior densidade, menor altura média e predomínio de indivíduos de espécies exigentes em luz. Do ponto de vista de conservação da biodiversidade da Floresta com Araucárias, o presente estudo sugere a necessidade da conservação de fragmentos cujo tamanho e configuração espacial permitam a existência de áreas de interior, com o propósito de contemplar a proteção de espécies não tolerantes às condições de borda.

Palavras-chave: Floresta Ombrófila Mista; fragmentos florestais; Planalto Catarinense.
\end{abstract}

\begin{abstract}
This study aimed to evaluate changes in the organization of tree species communities due to edge effects in a forest remnant in southern Brazil. Five transects were installed in the study area, perpendicular to the forest edge, with the dimensions of 100x20 m, subdivided into ten plots. The edge sector was defined for the distance of 0-50 m and the interior for the distance of 50-100 m. All trees with circumference at breast height $\geq 15.7$ were measured, identified and classified into regeneration guilds. The sectors were compared using the $t$ test for normally distributed variables, Mann-Whitney test for non-normal distributions, rarefaction, for richness, and Chi-square test applied to a contingency table, to check the distribution of individuals of different regeneration guilds. The sectorization as a function of edge and interior represented an important source of forest heterogeneity, once the most marginal part of the fragment was characterized by a tree component of higher density, smaller average height and prevalence of individuals of light demanding

1 Engenheiro Florestal, MSc., Departamento de Engenharia Florestal, Universidade do Estado de Santa Catarina, Av. Luiz de Camões, 2090, CEP 88520-000, Lages (SC), Brasil. francieli_pscheidt@hotmail.com / brunaflorestal@ yahoo.com.br / tiagoferreira@florestal.eng.br

2 Engenheiro Florestal, Dr., Professor do Departamento de Engenharia Florestal, Universidade do Estado de Santa Catarina, Av. Luiz de Camões, 2090, CEP 88520-000, Lages (SC), Brasil. higuchip@gmail.com / carol_sil4@ yahoo.com.br

3 . Engenheiro Agrônomo, Dr., Pesquisador da EPAGRI, Estação Experimental de Lages, CEP 88502-970, Lages (SC), Brasil.tassiodr@yahoo.com.br

4 Acadêmico de Engenharia Florestal, Departamento de Engenharia Florestal, Universidade do Estado de Santa Catarina, Av. Luiz de Camões, 2090, CEP 88520-000, Lages (SC), Brasil. marcelo.bonazza@hotmail.com / marcoanbento@gmail.com
\end{abstract}

Recebido para publicação em 23/04/2016 e aceito em 21/03/2017

Ci. Fl., v. 28, n. 2, abr. - jun., 2018 
species. In the context of biodiversity conservation of the Araucaria Forest, this study suggests the need for conservation of forest fragments which size and spatial configuration allow the existence of interior area, in order to contemplate the protection of non-tolerant species of edge conditions.

Keywords: Araucaria Forest; forest fragments; Santa Catarina plateau.

\section{INTRODUÇÃO}

O processo de fragmentação de habitat naturais pode ser considerado um dos principais problemas ecológicos da atualidade (LAURANCE et al., 2006; HADDAD et al., 2015; VALIENTE-BANUET et al., 2015), tendo como uma das consequências o surgimento do efeito de borda (MURCIA, 1995; TABARELLI; LOPES; PERES, 2008), que pode resultar em mudanças na estrutura e na composição das espécies na parte marginal dos remanescentes (WILLIAMS-LINERA; DOMÍNGUEZ-GASTELÚ; GARCÍA-ZURITA, 1998; LAURANCE et al., 2006). Dentre alterações provocadas pelo efeito de borda, destaca-se o aumento da luminosidade e temperatura na interface de transição entre a área florestal e a matriz não florestal, que ocasionam uma maior evapotranspiração e, consequentemente, a redução da disponibilidade de água (KAPOS, 1989; MURCIA, 1995). Assim, as alterações microclimáticas causadas por este processo atuam como filtros ambientais, selecionando espécies que são capazes de se instalar e se desenvolver nessa nova condição (MALCOLM, 1994). Como resultado, geralmente, o ecossistema fragmentado não consegue suportar o grupo de espécies encontradas apenas no ecossistema intacto (LAURANCE et al., 2002).

Nas áreas planálticas do Sul do Brasil, ainda pouco estudadas quanto ao tema (e.g. PSCHEIDT et al., 2015; FERREIRA et al., 2016), parte significativa da paisagem natural é caracterizada pela ocorrência de um mosaico composto por florestas com araucárias e campos. Como o clima atual, predominantemente úmido, favorece o desenvolvimento das florestas, estudos indicam que os campos representam um tipo de vegetação relictual de eras glaciais pretéritas (DÜMIG et al., 2008). De acordo com estudos palinológicos (BAUERMANN; BEHLING, 2009), a expansão do componente arbóreo sobre os campos se intensificou apenas recentemente, entre o Holoceno Médio e Superior, de 4320 a 1000 anos antes do presente. De fato, a expansão do componente florestal sobre os campos é frequentemente relatada na literatura, ocorrendo pelo avanço de bordas florestais e na forma de núcleos inseridos na matriz campestre (DUARTE et al., 2006; CARLUCCI et al., 2011). Porém, a partir do século XVII, a região foi palco de um processo de colonização histórico, que resultou em uma forte pressão sobre as áreas de florestas, por meio do desmatamento e distúrbios crônicos (e.g., corte seletivo e pecuária extensiva). Desta forma, no que se refere à dinâmica da paisagem, os fragmentos florestais regionais encontram-se sob influência de forças antagônicas: as condições climáticas que favorecem o avanço florestal sobre os campos e as pressões antrópicas que impedem esta expansão. Além disso, um longo histórico de perturbações nestas áreas pode ter resultado em um processo de homogeneização biológica, como já observado na região nordeste do Brasil (LÔBO et al., 2011), de forma que, em pequena escala espacial, não seria possível identificar diferenças florísticas-estruturais entre a borda e o interior dos remanescentes.

Desta forma, considerando que estudos nas áreas de interface entre campo e floresta são estratégicos para o entendimento da dinâmica da paisagem no contexto apresentado, procurou-se testar a hipótese de que existe variação da estrutura, diversidade, riqueza e participação de guildas de regeneração do componente arbóreo nos primeiros $100 \mathrm{~m}$ da faixa mais marginal de um fragmento florestal com araucárias no Sul do Brasil.

\section{MATERIAL E MÉTODOS}

O estudo foi realizado em um fragmento de Floresta Ombrófila Mista (FOM), situado em uma latitude de 27044'16,13" Sul e longitude de 50²8'51,35" Oeste, e altitude em torno de $900 \mathrm{~m}$, de propriedade da EPAGRI (Empresa de Pesquisa Agropecuária e Extensão Rural de Santa Catarina), localizado no município de São José do Cerrito, na região do Planalto Sul Catarinense. O fragmento tem uma área total de 710,60 ha e está inserido em uma matriz heterogênea, composta por pastagens, banhados e plantios silviculturais de Pinus spp. A área tem um histórico de perturbação comum na região, caracterizado pela substituição de áreas florestais por atividades pecuárias, e pelo corte seletivo de espécies no passado, com destaque para a 
extração de Araucária em meados do século passado. Após este período, com a criação do Código Florestal Brasileiro e regulamentações estaduais, a área está protegida da exploração. Desta forma, o fragmento se caracteriza por ser remanescente de um processo antigo de fragmentação ( $>50$ anos).

Para análise da vegetação, em áreas de matriz com pastagem, foram estabelecidas cinco transeções perpendiculares à borda, distanciadas, pelo menos, $100 \mathrm{~m}$ entre si, com a extensão de $100 \mathrm{~m}$ para o interior da floresta e a largura de $20 \mathrm{~m}$, totalizando 1 ha de área amostrada. Cada transeção foi subdividida em dez parcelas de $10 \times 20 \mathrm{~m}$, totalizando 50 parcelas. Foi considerado como setor borda as cinco primeiras parcelas $(0-50 \mathrm{~m})$ de cada transeção e como setor interior as cinco últimas (50-100 m). A extensão das transecções $(100 \mathrm{~m})$ e a subdivisão em borda e interior em $50 \mathrm{~m}$ foram consideradas pelo fato da forma dos fragmentos da região ser predominantemente irregular e a partir de revisões de literatura, em que, frequentemente, o efeito de borda sobre comunidades vegetais é relatado como sendo mais intenso nos primeiros 50 m (KAPOS, 1989; MURCIA, 1995).

Em cada parcela, todos os indivíduos arbóreos vivos que apresentaram CAP (circunferência a altura do peito, medida a 1,30 $\mathrm{m}$ do solo) igual ou superior a $15,7 \mathrm{~cm}$ foram identificados, marcados e tiveram seus CAPs mensurados e alturas estimadas. As espécies foram classificadas nas famílias organizadas de acordo com o sistema APG IV (ANGIOSPERM PHYLOGENY GROUP, 2016).

A suficiência amostral foi determinada por meio de curvas de acumulação de espécies, com a riqueza estimada por 1.000 permutações. Foi verificada a associação das espécies com cada setor (borda e interior) pela análise de espécies indicadoras, excluindo-se aquelas com menos do que cinco indivíduos. Foram calculados por setor: densidade, área basal, diâmetro médio, altura média, riqueza, diversidade (Índice de Shannon) e equabilidade (Índice de Pielou). O teste de Shapiro-Wilk foi utilizado para verificar a normalidade das variáveis. Para a comparação entre os setores, foi aplicado o teste t (significância com $p<0,05)$ para variáveis com a distribuição normal (densidade, área basal e diâmetro médio), o teste de Mann-Whitney (significância com $p<0,05$ ), para distribuições não normais (altura média, diversidade e equabilidade), e rarefação a partir de 500 indivíduos, para a comparação da riqueza. Para a análise da estrutura diamétrica e hipsométrica da comunidade nos setores, foram utilizados histogramas de frequência, com intervalos de classes (IC) determinados a partir da fórmula de Sturge. As distribuições dos indivíduos nas classes de tamanho foram comparadas entre os setores pelo teste de Kolmogorov-Smirnov.

As espécies amostradas foram classificadas em guildas de regeneração de acordo com Swaine e Whitmore (1988), modificado por Oliveira Filho et al. (1994), em: pioneiras (P), cujos indivíduos necessitam de luz direta para germinar e se estabelecer, clímax exigente de luz (CL), que precisam de luz quando jovens para atingir o dossel, e clímax tolerante à sombra (CS), que são as espécies cujas sementes germinam e que crescem sob a sombra, em condições de sub-bosque. A classificação das espécies foi baseada em revisão de literatura (e.g., SARAIVA, 2011; SILVA et al., 2013) e observações de campo. Na literatura, é frequente que algumas espécies apresentem divergência quanto à classificação em guildas de regeneração, como, por exemplo, Araucaria angustifolia (Bertol.) Kuntze e Myrsine coriacea (Sw.) Roem. \& Schult, encontradas tanto como pioneiras, quanto clímax exigentes em luz. Isto ocorre porque classificações artificias são tentativas de simplificar a grande complexidade que existe na natureza, de forma que muitos fatores podem atuar na capacidade ou não de uma espécie colonizar o dossel ou áreas abertas, como, por exemplo, a competição. Assim, no caso de divergências, nos baseamos em observações na área de estudo, como na presença de banco de plântulas dentro da floresta. Para a comparação das guildas entre os setores, foi aplicado um teste de qui-quadrado aplicado a uma tabela de contingência. Indivíduos não identificados ou identificados somente em nível de gênero não foram classificados e não participaram da análise.

A estrutura horizontal também foi descrita a partir do cálculo do Valor de Importância (VI) para cada espécie, calculada por setor. A determinação do VI foi segundo Mueller-Dombois e Ellemberg (1974), por meio da média da densidade relativa, frequência relativa e dominância relativa.

As análises dos dados foram realizadas utilizando a linguagem de programação estatística $R(R$ DEVELOPMENT CORE TEAM, 2016). Utilizou-se os pacotes Vegan (OKSANEN et al., 2016) e Labdsv (ROBERTS, 2016). 


\section{RESULTADOS E DISCUSSÃO}

A curva de acumulação (Figura 1) indicou que a suficiência amostral foi alcançada, pois, com um aumento de $10 \%$ na área amostral, o número de espécies aumentou apenas 2,58\%. Segundo Kersten e Galvão (2011), atinge-se a suficiência quando a curva tende à estabilidade e a adição de novas unidades amostrais não altera significativamente o número de espécies observadas, sendo sugerido que um aumento de $10 \%$ na área amostral resulte em um aumento de até $5 \%$ em novas espécies.

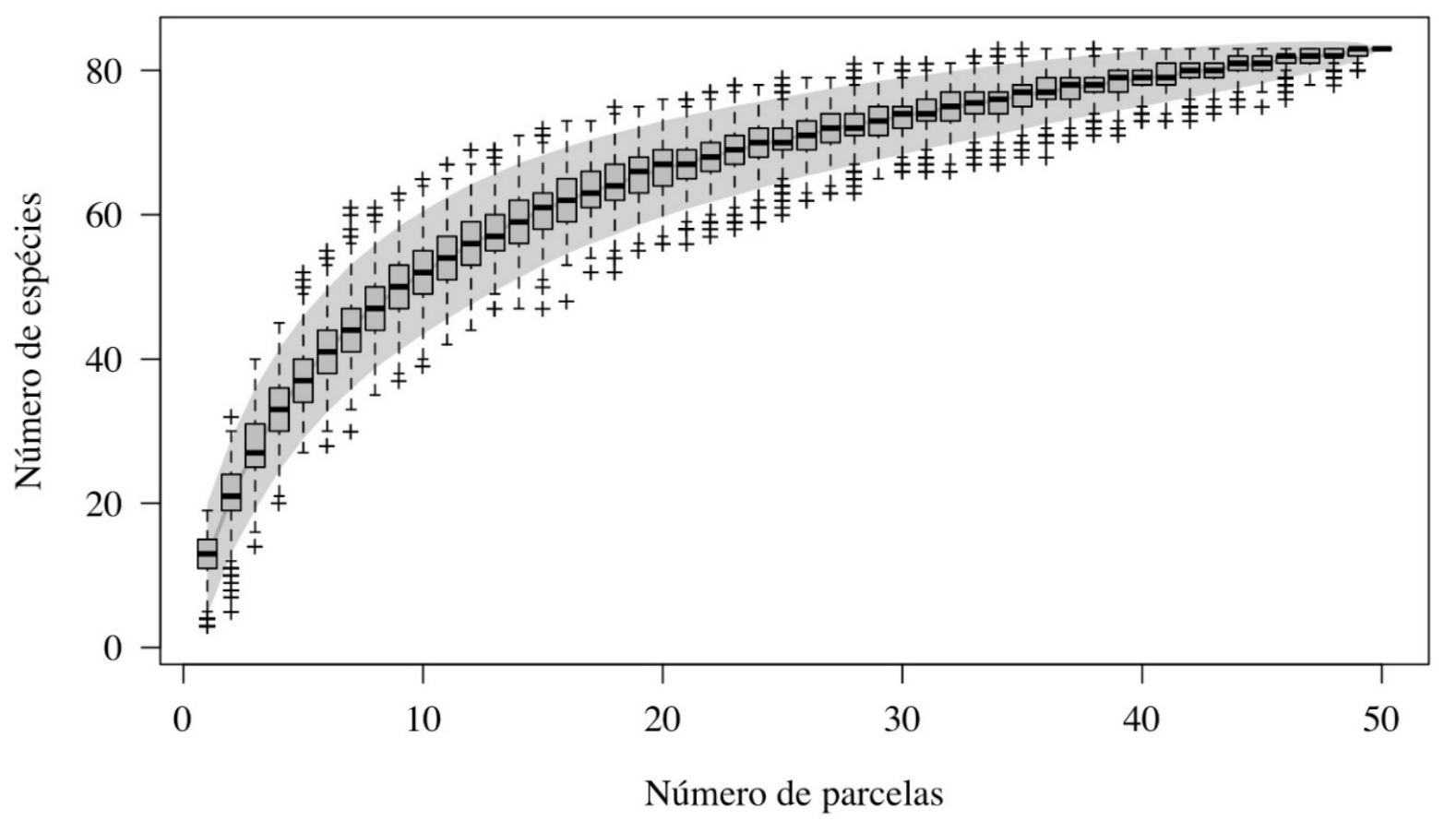

FIGURA 1: Curva de acumulação de espécies do fragmento de Floresta com Araucárias estudado no Planalto Catarinense, Sul do Brasil.

FIGURE 1: Species accumulation curve of an Araucaria Forest Fragment located in Santa Catarina plateau, southern Brazil.

No levantamento, foram encontrados 1.538 indivíduos, que foram classificados em 38 famílias, 62 gêneros e 82 espécies (Tabela 1), sendo dois indivíduos não identificados por ausência de folha na época do inventário. As famílias de maior riqueza específica foram Myrtaceae (17 espécies), seguida por Asteraceae (seis espécies), Lauraceae (cinco espécies), Salicaceae e Sapindaceae, cada uma com quatro espécies, Anacardiaceae, Aquifoliaceae, Euphorbiaceae, Fabaceae e Solanaceae, com três espécies cada. As 10 famílias citadas corresponderam a $62,7 \%$ da riqueza total. 
TABELA 1: Famílias e espécies, com suas respectivas guildas de regeneração (GR, sendo $\mathrm{P}=$ pioneiras; $\mathrm{CL}=$ clímax exigente de luz e $\mathrm{CS}=$ clímax tolerante à sombra) e números de indivíduos total $(\mathrm{N})$ e por setor borda $(\mathrm{Nb})$ e interior $(\mathrm{Ni})$, amostradas no componente arbóreo do fragmento de Floresta com Araucárias estudado no Planalto Catarinense, Sul do Brasil.

TABLE 1: Families and species, with their respective regeneration guilds (GR), being $\mathrm{P}=$ pioneers; $\mathrm{CL}=$ light demanding climax and $\mathrm{CS}=$ shade tolerant climax) and total number of individuals $(\mathrm{N})$ and number of individuals by sector, edge $(\mathrm{Nb})$ and interior $(\mathrm{Ni})$, sampled in the tree component of an Araucaria Forest Santa Catarina plateau, southern Brazil.

\begin{tabular}{|c|c|c|c|c|c|}
\hline Famílias & Espécies & $\mathrm{Nb}$ & $\mathrm{Ni}$ & $\mathrm{N}$ & GR \\
\hline \multirow[t]{3}{*}{ Anacardiaceae } & Lithrea brasiliensis Marchand & 32 & 32 & 64 & $\mathrm{P}$ \\
\hline & Schinus polygamus (Cav.) Cabrera & 1 & 0 & 1 & $\mathrm{P}$ \\
\hline & Schinus terebinthifolius Raddi & 3 & 3 & 6 & $\mathrm{P}$ \\
\hline Annonaceae & Annona rugulosa (Schltdl.) H.Rainer & 7 & 7 & 14 & $\mathrm{CL}$ \\
\hline \multirow[t]{3}{*}{ Aquifoliaceae } & Ilex microdonta Reissek & 1 & 0 & 1 & $\mathrm{CL}$ \\
\hline & Ilex paraguariensis A. St.-Hil. & 2 & 2 & 4 & $\mathrm{CS}$ \\
\hline & Ilex theezans Mart. ex Reissek & 2 & 3 & 5 & $\mathrm{CL}$ \\
\hline Araliaceae & Oreopanax fulvus Marchal & 0 & 1 & 1 & $\mathrm{CL}$ \\
\hline Araucariaceae & Araucaria angustifolia (Bertol.) Kuntze & 29 & 30 & 59 & $\mathrm{CL}$ \\
\hline Arecaceae & Butia eriospatha (Mart. ex Drude) Becc. & 1 & 0 & 1 & $\mathrm{P}$ \\
\hline \multirow[t]{6}{*}{ Asteraceae } & Baccharis semiserrata DC. & 1 & 0 & 1 & $\mathrm{P}$ \\
\hline & Baccharis uncinella DC. & 10 & 0 & 10 & $\mathrm{P}$ \\
\hline & Dasyphyllum spinescens (Less.) Cabrera & 13 & 6 & 19 & $\mathrm{P}$ \\
\hline & Gochnatia polymorpha (Less.) Cabr. & 0 & 1 & 1 & $\mathrm{P}$ \\
\hline & Symphyopappus lymansmithii B.L.Rob. & 1 & 0 & 1 & $\mathrm{P}$ \\
\hline & Vernonanthura discolor (Spreng.) H.Rob & 3 & 3 & 6 & $\mathrm{P}$ \\
\hline Bignoniaceae & Jacaranda puberula Cham. & 12 & 4 & 16 & $\mathrm{CL}$ \\
\hline Canellaceae & Cinnamodendron dinisii Schwanke & 26 & 16 & 42 & $\mathrm{CL}$ \\
\hline Cannabaceae & Celtis iguanaea (Jacq.) Sarg. & 1 & 0 & 1 & $\mathrm{P}$ \\
\hline Celastraceae & Maytenus muelleri Schwacke & 0 & 1 & 1 & $\mathrm{CS}$ \\
\hline Clethraceae & Clethra scabra Pers & 2 & 3 & 5 & $\mathrm{P}$ \\
\hline Cunoniaceae & Lamanonia ternata Vell. & 1 & 11 & 12 & $\mathrm{CL}$ \\
\hline Dicksoniaceae & Dicksonia sellowiana Hook. & 4 & 5 & 9 & $\mathrm{CS}$ \\
\hline Elaeocarpaceae & Sloanea monosperma Vell. & 0 & 1 & 1 & $\mathrm{CS}$ \\
\hline Erythroxylaceae & Erythroxylum deciduum A.St.-Hil. & 3 & 0 & 3 & $\mathrm{CL}$ \\
\hline Escalloniaceae & Escallonia bifida Link \& Otto & 1 & 0 & 1 & $\mathrm{P}$ \\
\hline \multirow[t]{3}{*}{ Euphorbiaceae } & Sapium glandulosum (L.) Morong & 6 & 1 & 7 & $\mathrm{P}$ \\
\hline & Sebastiania brasiliensis Spreng. & 1 & 0 & 1 & $\mathrm{CL}$ \\
\hline & Sebastiania commersoniana (Baill.) L.B.Sm. \& Downs & 17 & 1 & 18 & $\mathrm{CL}$ \\
\hline \multirow[t]{3}{*}{ Fabaceae } & Dalbergia frutescens (Vell.) Britton & 18 & 11 & 29 & $\mathrm{CL}$ \\
\hline & Inga virescens Benth. & 20 & 28 & 48 & $\mathrm{CL}$ \\
\hline & Mimosa scabrella Benth & 9 & 0 & 9 & $\mathrm{P}$ \\
\hline \multirow[t]{3}{*}{ Lauraceae } & Сinnатотит атоепит (Nees \& Mart.) Kosterm & 3 & 2 & 5 & CL \\
\hline & Nectandra lanceolata Nees & 17 & 3 & 20 & $\mathrm{CL}$ \\
\hline & Nectandra megapotamica (Spreng.) Mez & 22 & 6 & 28 & $\mathrm{CL}$ \\
\hline
\end{tabular}


TABELA 1: Continuação...

TABLE 1: Continued..

\begin{tabular}{|c|c|c|c|c|c|}
\hline Famílias & Espécies & $\mathrm{Nb}$ & $\mathrm{Ni}$ & $\mathrm{N}$ & GR \\
\hline & Ocotea puberula (Rich.) Nees & 23 & 11 & 34 & $\mathrm{CL}$ \\
\hline & Ocotea pulchella Mart & 19 & 24 & 43 & $\mathrm{CL}$ \\
\hline Loganiaceae & Strychnos brasiliensis (Spreng.) Mart. & 1 & 2 & 3 & $\mathrm{CS}$ \\
\hline Melastomataceae & Miconia cinerascens Miq. & 1 & 1 & 2 & $\mathrm{P}$ \\
\hline \multirow[t]{17}{*}{ Myrtaceae } & Acca sellowiana (O.Berg) Burret & 2 & 2 & 4 & $\mathrm{P}$ \\
\hline & Blepharocalyx salicifolius (Kunth) O.Berg & 6 & 3 & 9 & CL \\
\hline & Calyptranthes concinna DC. & 2 & 13 & 15 & $\mathrm{CS}$ \\
\hline & Campomanesia xanthocarpa O.Berg & 12 & 17 & 29 & $\mathrm{CL}$ \\
\hline & Eugenia pluriflora DC. & 1 & 4 & 5 & $\mathrm{CL}$ \\
\hline & Eugenia pyriformis Cambess. & 3 & 11 & 14 & CL \\
\hline & Myrcengenia euosma (O.Berg) D.Legrand & 2 & 5 & 7 & CL \\
\hline & Myrceugenia glaucescens (Cambess.) D.Legrand \& Kausel & 2 & 12 & 14 & $\mathrm{CS}$ \\
\hline & Myrceugenia oxysepala (Burret) D.Legrand \& Kausel & 0 & 1 & 1 & CL \\
\hline & Myrcia hatschbachii D. Legrand & 8 & 19 & 27 & $\mathrm{CS}$ \\
\hline & Myrcia selloi (Spreng.) N. Silveira & 1 & 2 & 3 & CL \\
\hline & Myrcia oblongata DC. & 17 & 10 & 27 & $\mathrm{P}$ \\
\hline & Myrcia palustris DC. & 1 & 3 & 4 & $\mathrm{CL}$ \\
\hline & Myrcia splendens (Sw.) DC. & 12 & 7 & 19 & CL \\
\hline & Myrcianthes gigantea (D.Legrand) D.Legrand & 0 & 1 & 1 & CL \\
\hline & Myrciaria delicatula (DC.) O.Berg & 3 & 1 & 4 & $\mathrm{CS}$ \\
\hline & Myrrhinium atropurpureum Schott & 1 & 1 & 2 & CL \\
\hline NI & Não identificadas & 0 & 2 & 2 & - \\
\hline Picramniaceae & Picramnia parvifolia Engl. & 0 & 1 & 1 & $\mathrm{CL}$ \\
\hline Podocarpaceae & Podocarpus lambertii Klotzsch ex Endl & 3 & 2 & 5 & $\mathrm{CL}$ \\
\hline \multirow[t]{2}{*}{ Primulaceae } & Myrsine coriacea (Sw.) Roem. \& Schult & 8 & 5 & 13 & $\mathrm{CL}$ \\
\hline & Myrsine umbellata Mart. & 5 & 4 & 9 & CL \\
\hline Proteaceae & Roupala montana Aubl. & 1 & 0 & 1 & $\mathrm{CS}$ \\
\hline Quillajaceae & Quillaja brasiliensis (A.St.-Hil. \& Tul.) Mart & 3 & 1 & 4 & $\mathrm{CL}$ \\
\hline \multirow[t]{2}{*}{ Rhamnaceae } & Rhamnus sphaerosperma $\mathrm{Sw}$ & 1 & 0 & 1 & $\mathrm{CL}$ \\
\hline & Scutia buxifolia Reissek & 1 & 0 & 1 & $\mathrm{CL}$ \\
\hline Rosaceae & Prunus myrtifolia (L.) Urb & 8 & 1 & 9 & $\mathrm{CL}$ \\
\hline \multirow[t]{2}{*}{ Rutaceae } & Zanthoxylum kleinii (R.S.Cowan) P.G.Waterman & 15 & 17 & 32 & $\mathrm{CL}$ \\
\hline & Zanthoxylum rhoifolium Lam & 12 & 1 & 13 & $\mathrm{CL}$ \\
\hline \multirow[t]{4}{*}{ Salicaceae } & Banara tomentosa Clos & 6 & 8 & 14 & $\mathrm{CL}$ \\
\hline & Casearia decandra Jacq. & 33 & 27 & 60 & $\mathrm{CS}$ \\
\hline & Casearia obliqua Spreng. & 0 & 5 & 5 & CL \\
\hline & Xylosma ciliatifolia (Clos) Eichler & 15 & 4 & 19 & $\mathrm{CL}$ \\
\hline \multirow[t]{4}{*}{ Sapindaceae } & Allophylus edulis (A.St.-Hil., Cambess. \& A.Juss.) Radlk & 47 & 15 & 62 & $\mathrm{CL}$ \\
\hline & Allophylus guaraniticus (A. St.-Hil.) Radlk. & 7 & 6 & 13 & $\mathrm{CS}$ \\
\hline & Cupania vernalis Cambess. & 44 & 44 & 88 & $\mathrm{CL}$ \\
\hline & Matayba elaeagnoides Radlk. & 247 & 151 & 398 & $\mathrm{CL}$ \\
\hline
\end{tabular}


TABELA 1: Continuação...

TABLE 1: Continued..

\begin{tabular}{llcccc}
\hline \multicolumn{1}{c}{ Famílias } & \multicolumn{1}{c}{ Espécies } & $\mathrm{Nb}$ & $\mathrm{Ni}$ & $\mathrm{N}$ & $\mathrm{GR}$ \\
\hline Solanaceae & Solanum mauritianum Scop. & 0 & 1 & 1 & $\mathrm{P}$ \\
& Solanum sanctaecatharinae Dunal & 5 & 9 & 14 & $\mathrm{P}$ \\
& Solanum sp. & 1 & 3 & 4 & - \\
\hline Styracaceae & Styrax leprosus Hook. \& Arn & 18 & 12 & 30 & $\mathrm{CL}$ \\
\hline Symplocaceae & Symplocos uniflora (Pohl) Benth & 4 & 1 & 5 & $\mathrm{CL}$ \\
\hline Verbenaceae & Citharexylum solanaceum Cham. & 7 & 9 & 16 & $\mathrm{P}$ \\
\hline Winteraceae & Drimys brasiliensis Miers & 0 & 1 & 1 & $\mathrm{CL}$ \\
\hline Total & & 877 & 661 & 1538 & \\
\hline
\end{tabular}

Das 51 espécies (desconsiderando-se as que apresentaram menos de cinco indivíduos), somente três foram indicadoras do ambiente em que estão situadas $(p<0,05)$ : Nectandra lanceolata Nees e Mimosa scabrella Benth foram indicadoras do setor de borda e Lamanonia ternata Vell. apresentou associação com o interior. Destaca-se que Mimosa scabrella apresentou todos os indivíduos na borda da floresta. Baccharis uncinella DC. e Sebastiania commersoniana (Baill.) L.B.Sm. \& Downs, apesar de apresentarem maior concentração de indivíduos na borda, não foram consideradas indicadoras deste setor, uma vez que apresentaram baixa frequência nas parcelas $(<17 \%)$, sendo que essa análise considera, além da densidade, a elevada frequência no setor para ser considerada indicadora. Segundo Murcia (1995), a criação dos efeitos de borda pode causar alterações na distribuição das espécies ao longo das bordas devido a suas diferentes tolerâncias fisiológicas. Portanto, infere-se que as espécies indicadoras foram as mais influenciadas pelos gradientes ambientais existentes da borda para o interior do fragmento.

Não foram encontradas diferenças significativas nos padrões de riqueza e diversidade e nos aspectos estruturais relacionados à área basal e diâmetro médio dos indivíduos (Tabela 2). Porém, observou-se que a borda apresentou significativamente maior densidade de indivíduos $(p=0,010)$, com menor altura média $(p=0,003)$. A presença de árvores de menor porte na borda foi reforçada pela análise da distribuição de indivíduos nas classes de tamanho de diâmetro e altura (Figura 2). Apesar do padrão de distribuição de frequência ser o mesmo na borda e no interior (diamétrica: $\mathrm{D}=0,07 ; p=1,00$; hipsométrica: $\mathrm{D}=0,27$; $p=0,66$ ), observa-se a tendência que na parte mais externa do fragmento ocorrem as maiores frequências de indivíduos nas classes de menores tamanhos de diâmetro e altura quando comparada com o interior. Ressalta-se que enquanto a distribuição diamétrica em todos os setores seguiu o padrão "J-invertido" (exponencial negativa), na qual é encontrada uma maior frequência de indivíduos nas classes de menor tamanho, na distribuição hipsométrica as classes centrais tenderam a apresentar maior abundância de indivíduos, padrões comuns em florestas naturais.

TABELA 2: Densidade, área basal, diâmetro médio, altura média, riqueza, diversidade e equabilidade nos setores borda e interior do fragmento de Floresta com Araucárias estudado no Planalto Catarinense, Sul do Brasil.

TABLE 2: Density, basal area, average diameter, average height, richness, diversity and evenness sectors in the edge and interior of an Araucaria Forest fragment in Santa Catarina plateau, southern Brazil.

\begin{tabular}{lccc}
\hline & Borda & Interior & $p$ \\
\hline Densidade (ind./ha) & 1754 & 1322 & 0,010 \\
Área basal $\left(\mathrm{m}^{2} / \mathrm{ha}\right)$ & 35,86 & 32,30 & 0,444 \\
Diâmetro médio (cm) & 13,44 & 14,58 & 0,134 \\
Altura média (m) & 9,20 & 11,00 & 0,003 \\
Riqueza por rarefação $(\mathrm{n}=500)$ & $61,78(2,48)$ & $64,07(1,92)$ & \\
Diversidade (H') & 3,26 & 3,38 & 0,788 \\
Equabilidade (J) & 0,76 & 0,80 & 0,788 \\
\hline
\end{tabular}

Em que: Valores entre parênteses para riqueza por rarefação representam erros padrão do número de espécies e o valor de $p$ representa a significância pelo teste $\mathrm{t}$ (densidade, área basal e diâmetro médio) e Mann Whitney (altura média, diversidade e equabilidade). 


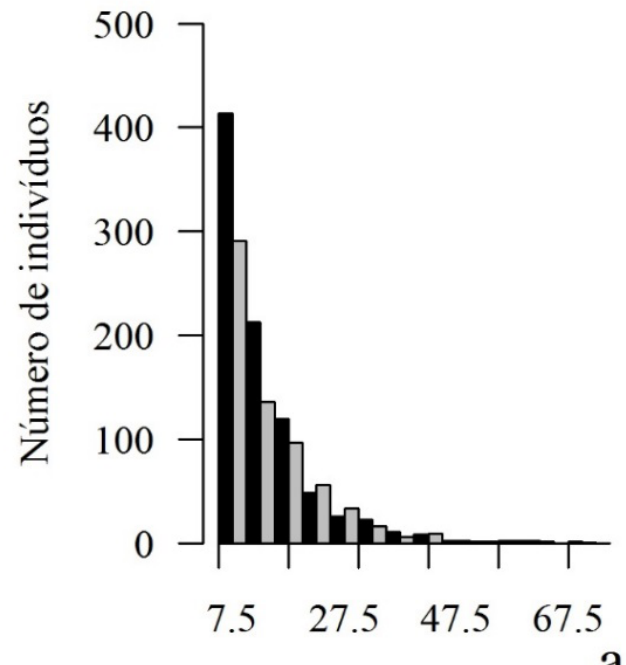

Classes de diâmetro

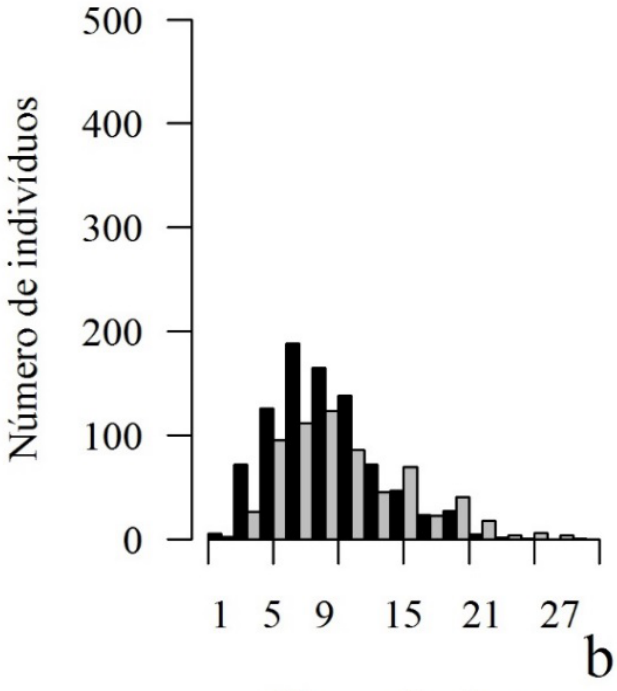

Classes de altura

FIGURA 2: Estrutura diamétrica (a) e hipsométrica (b) da comunidade de espécies arbóreas amostrada nos setores borda (barras pretas) e interior (barras cinzas) do fragmento de Floresta com Araucárias estudado no Planalto Catarinense, Sul do Brasil.

FIGURE 2: Diametric ( $a$ and $b$ ) and hypsometric ( $c$ and d) structure of tree species community sampled in border (black bars) and the interior (gray bars) sectors in an Araucaria Forest fragment in Santa Catarina plateau, southern Brazil.

$\mathrm{Na}$ análise da distribuição dos indivíduos pertencentes às guildas de regeneração também houve a existência de associação significativa $(\chi 2=17,059, p<0,0002)$ das mesmas com a borda e o interior (Tabela $3)$, reforçando a distinção entre os setores. As pioneiras e climácicas exigentes em luz ocorreram preferencialmente na borda e as climácicas tolerantes à sombra ocorreram preferencialmente no interior.

TABELA 3: Número de indivíduos pertencentes a cada guilda de regeneração do fragmento de Floresta com Araucárias estudado no Planalto Catarinense, Sul do Brasil.

TABLE 3: Number of individuals belonging to each guild regeneration in an Araucaria Forest in Santa Catarina plateau, southern Brazil.

\begin{tabular}{lcc} 
& Borda & Interior \\
\cline { 2 - 3 } Clímax exigente em luz & $697(676,4)$ & $486(506,6)$ \\
Pioneiras & $116(112,6)$ & $81(84,4)$ \\
Clímax tolerante à sombra & $63(86,9)$ & $89(65,1)$ \\
\hline
\end{tabular}

Valores entre parênteses representam o número de individuos esperado, de acordo com uma distribuição regular, pelo teste de qui-quadrado $\left(\chi^{2}=17,059, p<0,0002\right)$.

Desta forma, os resultados evidenciam que a floresta apresenta uma variação estrutural e funcional associada aos setores de distância da borda, com a parte mais periférica do fragmento apresentando um componente arbóreo de menor porte, com maior densidade de indivíduos e ocorrência preferencial de espécies exigentes em luz, como M. scabrella, o que sugere um estágio de sucessão florestal mais inicial neste setor. Estes resultados indicam que a setorização utilizada foi adequada para a avaliação do efeito de borda no fragmento estudado, de forma que as observações realizadas estão de acordo com a literatura existente, que indica efeitos de borda intensos nos 50 m marginais dos fragmentos (MURCIA, 1995). Este é um padrão comumente observado em fragmentos florestais com históricos de perturbação distintos, o que reforça a relevância das diferenças ambientais entre a borda e o interior de fragmentos como condicionantes de comunidades de espécies arbóreas. Tabarelli, Lopes e Peres (2008), em uma revisão sobre o tema, indicaram que a predominância de uma assembleia dominada por espécies pioneiras nas bordas não representa um 
estágio sucessional transitório, mas sim uma condição semelhante à final em estagnação. Já em áreas onde o processo de fragmentação é mais antigo (> 30 anos), como no presente caso, variações estruturais e na distribuição das guildas de regeneração podem estar relacionadas, também, com a expansão da área florestal sobre áreas de campos ou pastagens abandonadas, como relatado por Oosterhoorn e Kappelle (2000) em uma floresta nebular na Costa Rica, e por Carlucci et al. (2011), no Sul do Brasil.

No que se refere às espécies com os maiores Valores de Importância (VI), observa-se que o componente arbóreo apresentou elevada semelhança entre os setores de borda $(0-50 \mathrm{~m})$ e interior $(50-100 \mathrm{~m})$, com um compartilhamento de sete espécies (Matayba elaeagnoides Radlk., Ocotea puberula (Rich.) Nees, Lithrea brasiliensis Marchand, Araucaria angustifolia, Cupania vernalis Cambess, Ocotea pulchella Mart e Casearia decandra Jacq.) dentre as dez de maior importância relativa (Figura 3). Tanto na borda quanto no interior, Matayba elaegnoides foi a espécie de maior VI. Já Araucaria angustifolia, usualmente observada como sendo a de maior representatividade na Floresta Ombrófila Mista, ocupou apenas a quarta posição dentre as mais importantes em ambos setores. Neste caso, de acordo com o histórico conhecido da área, a baixa representatividade de Araucaria angustifolia pode estar relacionada com o corte seletivo no passado. A representativa participação das espécies citadas é um padrão reportado em vários estudos realizados em fragmentos de Floresta Ombrófila Mista no Sul do Brasil (e.g. HIGUCHI et al., 2012; SILVA et al., 2012; GASPER et al., 2013).
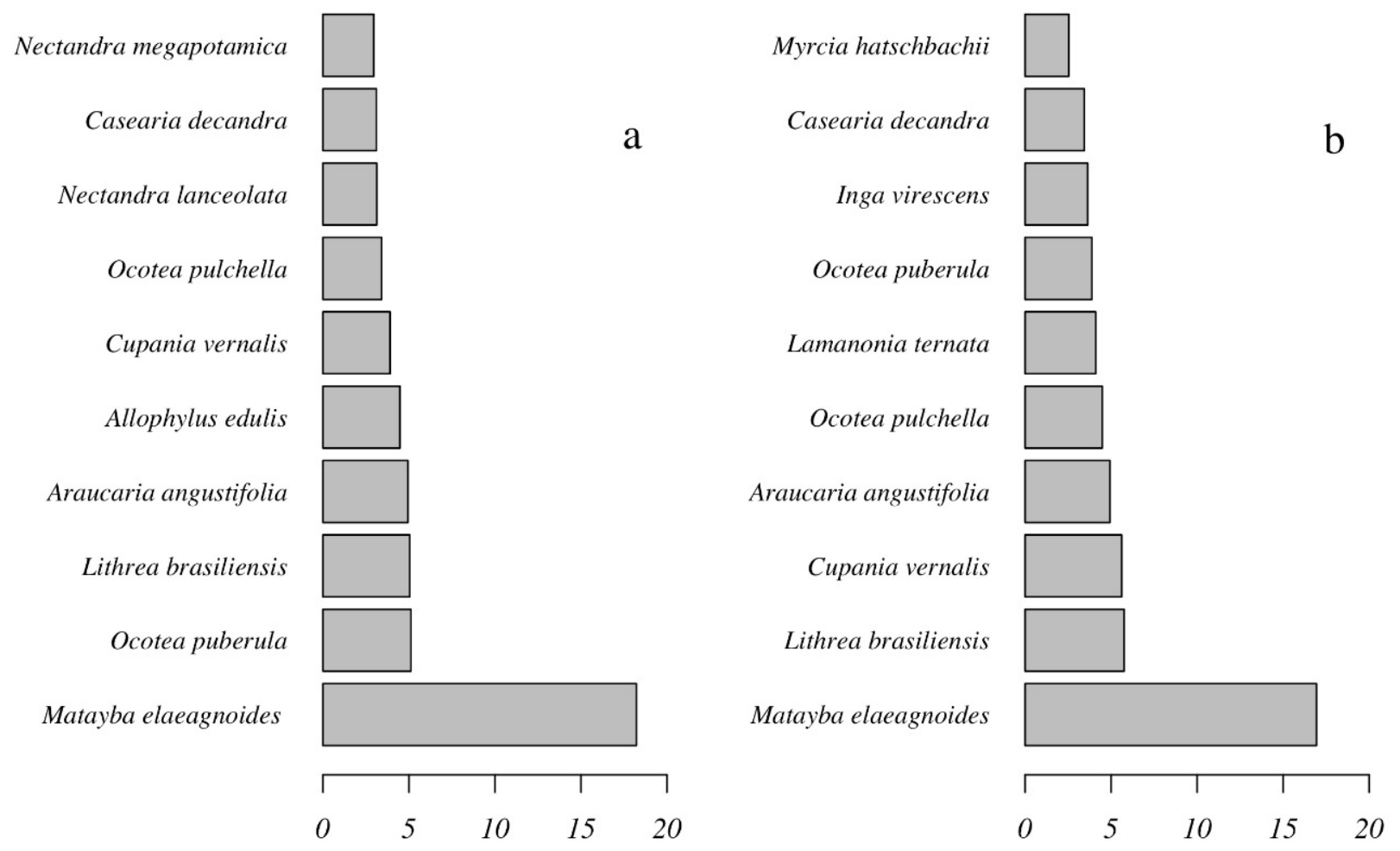

FIGURA 3: Valor de importância das 10 espécies de maior importância no setor borda (a) e no setor interior (b) do fragmento de Floresta com Araucárias estudado no Planalto Catarinense, Sul do Brasil.

FIGURE 3: Importance values of 10 most important species in edge sector (a) and in interior sector (b) in an Araucaria Forest, in Santa Catarina plateau, southern Brazil.

Apesar da similaridade quanto às espécies mais representativas, foi possível identificar a ocorrência preferencial de algumas espécies por setor, com Myrcia hatschbachii D. Legrand, Inga virescens Benth. e Lamanonia ternata (esta última indicadora de interior, de acordo com a Análise de Espécies Indicadoras) dentre as de maior VI no interior, e Allophylus edulis (A.St.-Hil., Cambess. \& A. Juss.), Nectandra megapotamica Nees e Nectandra lanceolata (esta última indicadora de borda, segundo a Análise de Es- 
pécies Indicadoras) como as de maior VI na borda. Esse padrão pode ser reflexo de uma menor amplitude de nicho ecológico dessas espécies diante dos gradientes ambientais existentes em função da distância da borda. Dentre os fatores ambientais que mais têm sido relatados quanto à variação em função da distância da borda, destaca-se o microclima. Nas bordas (e.g. maior abertura do dossel, maior temperatura do solo) (WILLIAMS-LINERA; DOMÍNGUEZ-GASTELÚ; GARCÍA-ZURITA, 1998), as condições podem ser limitantes para o desenvolvimento de espécies exigentes em sombra, causando maior mortalidade de indivíduos deste grupo neste setor (LAURANCE et al., 2006; PÜTZ et al., 2011). Os resultados relativos à ocorrência preferencial de indivíduos de espécies exigentes em luz na borda e tolerantes ao sombreamento no interior (Tabela 2) corroboram este gradiente microclimático no fragmento estudado.

Desta forma, infere-se que mesmo em fragmentos com um histórico antigo de fragmentação e perturbação, como aqueles de Floresta Ombrófila Mista no Sul do Brasil, o efeito de borda pode ser um importante condicionante para a distribuição das espécies. Esse resultado confirma as observações encontradas por Pscheidt et al. (2015) em outro fragmento florestal da região, também classificado como de Floresta Ombrófila Mista. Assim, em um cenário de possível processo de homogeneização biológica, como aquele já relatado em áreas de Floresta Atlântica na região Nordeste (LÔBO et al., 2011), supõe-se que os grupos formados pelas espécies de ocorrência preferencial nas bordas e indiferentes quanto a esta setorização, poderiam aumentar ainda mais a sua dominância nas áreas com florestas fragmentadas e sujeitas a distúrbios antrópicos crônicos, à custa daquelas de ocorrência preferencial no interior.

\section{CONCLUSÕES}

Conclui-se que o efeito de borda representou uma importante fonte de alteração do componente arbóreo para um fragmento de Floresta com Araucárias no Sul do Brasil, com um histórico de fragmentação e perturbações antrópicas crônicas. Na área mais externa do fragmento, na interface com locais de pastagens, observou-se uma assembleia de espécies arbóreas em fase mais inicial de sucessão florestal e de menor porte. Do ponto de vista de conservação da biodiversidade da Floresta com Araucárias, o presente estudo indica a necessidade da conservação de fragmentos com tamanho e forma que permita a existência de uma área de interior não sujeita ao efeito de borda.

\section{AGRADECIMENTOS}

À FUMDES/SC, pela bolsa de mestrado concedida à primeira autora, ao CNPq, pelo financiamento do projeto, por meio do Edital MCT/CNPq 14/2010, processo 475095/2010-3, e pelas bolsas de produtividade concedida ao segundo e terceiro autores. A Empresa de Pesquisa Agropecuária e Extensão Rural de Santa Catarina (EPAGRI), por gentilmente ter permitido acesso à área de sua propriedade.

\section{REFERÊNCIAS}

ANGIOSPERM PHYLOGENY GROUP. An update of the Angiosperm Phylogeny Group classification for the orders and families of flowering plants: APG IV. Botanical Journal of the Linnean Society, Londres, v. 181, n. 1, p. 1-20, 2016.

BAUERMANN, S. G.; BEHLING, H. Dinâmica paleovegetacional da Floresta com Araucária a partir do final do Pleistoceno: o que mostra a palinologia. In: FONSECA, C. R. et al. (Org.). Floresta com Araucária: ecologia, conservação e desenvolvimento. Ribeirão Preto: Holos, 2009. p. 35-38. CARLUCCI, M. B. et al. Edge expansion of Araucaria forest over southern Brazilian grasslands relies on nurse plant effect. Community Ecology, Budapeste, v. 12, n. 2, p. 196-201, 2011.

DUARTE, L. S. et al. Role of nurse plants in Araucaria Forest expansion over grassland in south Brazil. Austral Ecology, Malden, v. 31, n. 4, p. 520-528, 2006.

DÜMIG, A. et al. Araucaria forest expansion on grassland in the southern Brazilian highlands as revealed by $14 \mathrm{C}$ and $\delta 13 \mathrm{C}$ studies. Geoderma, Amsterdam, v. 145, n. 1, p. 143-157, 2008.

FERREIRA, T. S. et al. Composição florístico-estrutural ao longo de um gradiente de borda em fragmento de Floresta Ombrófila Mista Alto-Montana em Santa Catarina. Ciência Florestal, Santa Maria, 
v. 26, n. 1, p. 123-134, 2016.

GASPER, A. L. et al. Inventário florístico florestal de Santa Catarina: espécies da Floresta Ombrófila Mista. Rodriguésia, Rio de Janeiro, v. 64, n. 2, p. 201-210, 2013.

HADDAD, N. M. et al. Habitat fragmentation and its lasting impact on Earth's ecosystems. Science Advances, Washington, v. 1, n. 2, p. 1-9, 2015.

HIGUCHI, P. et al. Floristic composition and phytogeography of the tree component of Araucaria Forest fragments in southern Brazil. Brazilian Journal of Botany, São Paulo, v. 35, n. 2, p. 145-157, 2012.

KAPOS, V. Effects of isolation on the water status of forest patches in the Brazilian Amazon. Journal of Tropical Ecology, Cambridge, v. 5, n. 2, p. 173-185, 1989.

KERSTEN, R. A.; GALVÃO, F. Suficiência amostral em inventários florísticos e fitossociológicos. In: FELFILI, J. M. et al. (Org.). Fitossociologia no Brasil: métodos e estudos de casos. Viçosa, MG: Editora UFV, 2011. p. 156-173.

LAURANCE, W. F. et al. Ecosytem decay of Amazonian Forest fragments: a 22-year investigation. Conservation Biology, Hoboken, v. 16, n. 3, p. 605-618, 2002.

LAURANCE, W. F. et al. Rapid decay of tree-community composition in Amazonian forest fragments. Proceedings of the National Academy of Sciences, Washington, v. 103, n. 50, p. 19010-19014, 2006.

LÔBO, D. et al. Forest fragmentation drives Atlantic forest of northeastern Brazil to biotic homogenization. Diversity and Distributions, Malden, v. 17, n. 2, p. 287-296, 2011.

MALCOLM, J. R. Edge effects in Central Amazonian forest fragments. Ecology, Ithaca, v. 75, n. 8, p. 2438-2445, 1994.

MUELLER-DOMBOIS, D.; ELLENBERG, H. Aims and methods of vegetation ecology. New York: Wiley, 1974. $547 \mathrm{p}$.

MURCIA, C. Edge effects in fragmented forest: implications for conservation. Trends in Ecology \& Evolution, Londres, v. 10, n. 2, p. 58-62, 1995.

OKSANEN, J. et al. Vegan: Community Ecology Package. Disponível em: <http://cran.r-project.org/ package=vegan $>$. Acesso em: 15 jan. 2016.

OLIVEIRA FIHO, A. T. et al. Effects of soils and topography on the distribution of tree species in a tropical riverine forest in south-eastern Brazil. Journal of Tropical Ecology, Cambridge, n. 4, v. 10, p. 483-508, 1994.

OOSTERHOORN, M.; KAPPELLE, M. Vegetation structure and composition along an interior-edgeexterior gradient in a Costa Rican montane cloud forest. Forest Ecology and Management, Amsterdam, v. 126, n. 3, p. 291-307, 2000.

PSCHEIDT, F. et al. Variações florístico-estruturais da comunidade arbórea associadas à distância da borda em um fragmento florestal no Planalto Sul Catarinense. Floresta, Curitiba, v. 45, n. 2, p. 421-430, 2015.

PÜTZ, S. et al. Fragmentation drives tropical forest fragments to early successional states: a modelling study for Brazilian Atlantic forests. Ecological Modelling, Amsterdam, v. 222, n. 12, p. 1986-1997, 2011. R DEVELOPMENT CORE TEAM. R: a language and environment for statistical computing. [2016]. Disponível em: <http://www.R-project.org> Acesso em: 15 jan. 2016.

ROBERTS, D. W. labdsv: ordination and multivariate analysis for ecology. [2016]. Disponível em: $<$ http://CRAN.R-project.org/package=labdsv> Acesso em: 15 jan. 2016.

SARAIVA, D. D. Composição e estrutura de uma floresta ribeirinha no sul do Brasil. Biotemas, Florianópolis, v. 24, n. 4, p. 49-58, 2011.

SILVA, A. C. et al. Caracterização fitossociológica e fitogeográfica de um trecho de floresta ciliar em Alfredo Wagner, SC, como subsídio para restauração ecológica. Ciência Florestal, Santa Maria, v. 23, n. 4, p. 579-593, 2013.

SILVA, A. C. et al. Relações florísticas e fitossociologia de uma Floresta Ombrófila Mista Montana secundária em Lages, Santa Catarina. Ciência Florestal, Santa Maria, v. 22, n. 1, p. 193-206, 2012.

SWAINE, M. D.; WHITMORE, T. C. On the definition of ecological species groups in tropical rain forests. Plant Ecology, Dordrecht, v. 75, n. 1-2, p. 81-86, 1988.

TABARELLI, M.; LOPES, A. V.; PERES, C. A. Edge-effects drive tropical forest fragments towards an early-successional system. Biotropica, Malden, v. 40, n. 6, p. 657-661, 2008.

VALIENTE $\square$ BANUET, A. et al. Beyond species loss: the extinction of ecological interactions in a changing

Ci. Fl., v. 28, n. 2, abr. - jun., 2018 
world. Functional Ecology, Hoboken, v. 29, n. 3, p. 299-307, 2015.

WILLIAMS-LINERA, G.; DOMÍNGUEZ-GASTELÚ, V.; GARCÍA-ZURITA, M. E. Microenvironment and floristics of different edges in a fragmented tropical rainforest. Conservation Biology, Hoboken, v. 12, n. 5, p. 1091-1102, 1998. 